\title{
An echocardiographic assessment of cardiac morphology and common ECG findings in teenage professional soccer players: reference ranges for use in screening
}

\author{
J D Somauroo, J R Pyatt, M Jackson, R A Perry, D R Ramsdale
}

\begin{abstract}
Objective-To assess physiological cardiac adaptation in adolescent professional soccer players. Subjects and design-Over a 32 month period 172 teenage soccer players were screened by echocardiography and ECG at a tertiary referral cardiothoracic centre. They were from six professional soccer teams in the north west of England, competing in the English Football League. One was excluded because of an atrial septal defect. The median age of the 171 players assessed was 16.7 years (5th to 95th centile range: 14-19) and median body surface area $1.68 \mathrm{~m}^{2}$ $\left(1.39-2.06 \mathrm{~m}^{2}\right)$.

Main outcome measures-Standard echocardiographic measurements were compared with predicted mean, lower, and upper limits in a cohort of normal controls after matching for age and surface area. Univariate regression analysis was used to assess the correlation between echocardiographic variables and the age and surface area of the soccer player cohort. ECG findings were also assessed.

Results-All mean echocardiographic variables were greater than predicted for age and surface area matched controls $(\mathrm{p}<0.001)$. All variables except left ventricular septal and posterior wall thickness showed a modest linear correlation with surface area $(r=0.2$ to $0.4, \mathrm{p}<0.001)$; however, left ventricular mass was the only variable that was significantly correlated with age $(r=0.2$, $\mathrm{p}<0.01)$. Only six players $(3.5 \%)$ had structural anomalies, none of which required further evaluation. All had normal left ventricular systolic function. Sinus bradycardia was found in 65 $(39 \%)$. The Solokow-Lyon voltage criteria for left ventricular hypertrophy were present in 85 $(50 \%)$ and the Romhilt-Estes points score (five or more) in 29 (17\%). Repolarisation changes were present in $19(11 \%)$, mainly in the inferior leads.

Conclusions-Chamber dimensions, left ventricular wall thickness and mass, and aortic root size were all greater than predicted for controls after matching for age and surface area. Sinus bradycardia and the ECG criteria for left ventricular hypertrophy were common but there was poor correlation with echocardiographic left ventricular hypertrophy. The type of hypertrophy found reflected the combined endurance and strength based training undertaken.

(Heart 2001;85:649-654)
\end{abstract}

Keywords: cardiac morphology; professional soccer players; echocardiography; ECG findings

Regular physical training in athletes causes adaptive structural and functional changes within the heart. ${ }^{1}$ These consist principally of cardiac hypertrophy and a resting bradycardia, the clinical manifestations of which are known as "athlete's heart." The accompanying echocardiographic and ECG changes depend on the type of exercise undertaken. More isotonic forms of exercise found in endurance sports, such as long distance running and swimming, tend to cause an increase in ventricular chamber dimensions, but with only mild but proportional increases inwall thickness. In contrast, isometric exercise, found in the predominantly strength trained sports of weight lifting and wrestling, tend to increase wall thickness alone. ${ }^{2-5}$ The ECG of athletes regularly shows sinus bradycardia and sinus arrhythmia, left ventricular hypertrophy on voltage criteria, and depolarisation and repolarisation changes, especially in the anterior leads. This often simulates heart disease, with resultant diagnostic confusion. ${ }^{6}$
Adult elite professional footballers, who undertake both isotonic and isometric forms of exercise, have been found to have increased wall thickness, chamber dimensions, aortic root size, and left ventricular mass compared with healthy controls, ${ }^{7}$ but there are limited data on adolescent players. Such changes are important as they may simulate heart disease and cause inadvertent labelling and anxiety among soccer players and health professionals, with possible exclusion from sport. Our aim was to define the echocardiographic changes in teenage professional soccer players, to assess important ECG findings, and to characterise the type of cardiac morphological adaptation associated with their training.

\section{Methods}

SUBJECTS

Young soccer players from six professional teams in the north west of England competing in the English Football League have been routinely screened for unsuspected cardiovascular disease at our tertiary referral centre. 
Table 1 Characteristics of the 171 adolescent professional soccer players

\begin{tabular}{lcc}
\hline & \multicolumn{2}{c}{$\begin{array}{l}\text { 5th to 95th centile } \\
\text { range }\end{array}$} \\
\hline Age (years) & 16.7 & $15.3-18.3$ \\
Height $(\mathrm{cm})$ & 177.9 & $170.3-188.1$ \\
Weight $(\mathrm{kg})$ & 68.4 & $57.2-80.6$ \\
Body surface area $\left(\mathrm{m}^{2}\right)$ & 1.68 & $1.39-2.06$ \\
\hline
\end{tabular}

Over a 32 month period, 172 soccer players were screened. Their age, weight $(\mathrm{kg})$, and height $(\mathrm{cm})$ were recorded. The body surface area (BSA) was calculated according to the formula ${ }^{8}$ :

BSA $\left(\mathrm{m}^{2}\right)=(0.0001)(71.84)\left(\right.$ weight $\left.^{0.425}\right)\left(\right.$ height $\left.^{0.725}\right)$

\section{ECHOCARDIOGRAPHIC ASSESSMENT}

Transthoracic echocardiographic examinations were obtained in all subjects in the left lateral decubitus position using a Hewlett-Packard Sonos 5500 ultrasound system, with a $2.5 \mathrm{MHz}$ transducer (Hewlett-Packard Inc, Andover, Massachusetts, USA). Both M mode and cross sectional studies were performed. The $M$ mode echocardiogram of the left ventricle was recorded at the level of the tips of the mitral valve using the two dimensional image to obtain the optimal position and angulation of the $M$ mode line. Measurements of the left atrial and aortic root end systolic dimension, right ventricular end diastolic dimension (RVDd), left ventricular end diastolic posterior wall dimension (LVPWd), interventricular septal end diastolic dimension (IVSd), left ventricular end systolic internal dimension (LVIDs), and left ventricular end diastolic internal dimension (LVIDd) were calculated in the parasternal long axis view using the recommendations of the American Society of Echocardiography. ${ }^{9}$ Ejection fraction was calculated by cross sectional echocardiography. ${ }^{10}$ Left ventricular mass was calculated by the Penn convention and the American Society of Echocardiographers' convention using the following equations ${ }^{11}{ }^{12}$ :

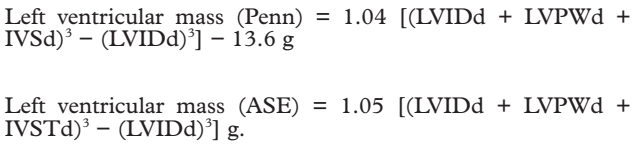

For the control data, the mean and the lower and upper limits of normal were derived by prediction using both age and surface area equations. ${ }^{13}$ Mean dimensions for the soccer players were compared with those predicted for age and surface area matched controls. The number $(\%)$ of soccer players who exceeded the upper limit of the normal ranges was also calculated.

\section{ECG EVALUATION}

Standard 12 lead surface ECGs were obtained in all subjects when rested and in the supine position, using an analogue system (Marquette Electronics, Milwaukee, Wisconsin, USA). The paper speed was $25 \mathrm{~mm} / \mathrm{s}$ with a $10 \mathrm{~mm} /$ $\mathrm{mV}$ gain and a $40 \mathrm{~Hz}$ low pass filter setting. Recordings were analysed by an experienced observer (JRP) blinded to the clinical details. Measurements were taken using calipers and a millimetre ruler. The following ECG variables were assessed: heart rate, $P$ axis, $Q R S$ axis, $T$ axis, PR interval, QRS duration, QTc interval, $P$ wave voltage and morphology, ST segment depression, and $\mathrm{T}$ wave inversion (repolarisation changes). Left axis deviation was defined as a QRS duration greater than $-30^{\circ}$, and right axis deviation as a QRS duration greater than $+120^{\circ}$. The QT intervals were corrected for heart rate (QTc) using Bazett's formula. ${ }^{14} \mathrm{P}$ wave voltage was assessed in lead V1, and right atrial enlargement was defined as a $\mathrm{P}$ wave voltage of $0.25 \mathrm{mV}$ or above. ${ }^{15}$ Left ventricular hypertrophy was defined by the Solokow-Lyon voltage criteria ${ }^{16}$ and the Romhilt-Estes points score system, a score of 5 or more indicating left ventricular hypertrophy. ${ }^{17}$ Right ventricular hypertrophy was assessed by the SolokowLyon voltage criteria. ${ }^{18}$ Pathological Q waves were defined as greater than 0.04 seconds in duration or more than $25 \%$ of the height of the $\mathrm{R}$ wave, or both. The presence of any further electrical abnormalities, such as prominent $U$ waves, delta waves, or a dominant $\mathrm{R}$ wave in V1, was also noted.

STATISTICAL ANALYSIS

Continuous data are expressed as mean (SD), with 5th to 95th centile ranges to facilitate comparison with data predicted for controls matched for age and surface area. Discrete

Table 2 Echocardiographic results: comparison between adolescent professional soccer players and normal controls matched for age and body surface area

\begin{tabular}{|c|c|c|c|c|c|c|c|c|}
\hline Measurement & $\begin{array}{l}\text { PSP: mean } \\
(S D)^{*}\end{array}$ & $\begin{array}{l}\text { PSP: 5th to } \\
95 \text { th centile } \\
\text { range }\end{array}$ & $\begin{array}{l}\text { Age matched } \\
\text { normal } \\
\text { controls: mean } \\
(S D)\end{array}$ & $\begin{array}{l}\text { Age matched } \\
\text { normal controls: } \\
\text { 5th to 95th centile } \\
\text { range }\end{array}$ & $\begin{array}{l}\text { PSP: number (\%) } \\
\text { greater than upper } \\
\text { limit of age matched } \\
\text { controls }\end{array}$ & $\begin{array}{l}\text { BSA matched } \\
\text { normal controls: } \\
\text { mean }(S D)\end{array}$ & $\begin{array}{l}\text { BSA matched } \\
\text { normal controls: } \\
\text { 5th to 95th centile } \\
\text { range }\end{array}$ & $\begin{array}{l}\text { PSP: number (\%) } \\
\text { greater than upper } \\
\text { limit of BSA } \\
\text { matched normal } \\
\text { controls }\end{array}$ \\
\hline RVDd $(\mathrm{cm})$ & $2.08(0.38)$ & $1.55-2.66$ & $1.47(0.02)$ & $0.92-2.02$ & $94(55 \%)$ & $1.07(0.01)$ & $0.98-1.62$ & $150(88 \%)$ \\
\hline IVSd $(\mathrm{cm})$ & $1.03(0.14)$ & $0.80-1.29$ & $0.83(0.01)$ & $0.63-1.03$ & $81(47 \%)$ & $0.90(0.06)$ & $0.71-1.09$ & $52(30 \%)$ \\
\hline LVIDd (cm) & $5.14(0.39)$ & $4.44-5.73$ & $4.74(0.10)$ & $4.20-5.29$ & $65(38 \%)$ & $4.94(0.34)$ & $4.42-5.47$ & $27(16 \%)$ \\
\hline LVPWd $(\mathrm{cm})$ & $1.00(0.15)$ & $0.74-1.24$ & $0.82(0.02)$ & $0.63-1.02$ & $75(44 \%)$ & $0.86(0.07)$ & $0.68-1.05$ & $67(39 \%)$ \\
\hline LVIDs (cm) & $3.39(0.41)$ & $2.76-4.01$ & $3.00(0.06)$ & $2.54-3.46$ & $83(49 \%)$ & $3.15(0.22)$ & $2.70-3.60$ & $51(30 \%)$ \\
\hline Ao root $(\mathrm{cm})$ & $2.92(0.29)$ & $2.50-3.41$ & $2.38(0.05)$ & $2.01-2.74$ & $126(74 \%)$ & $2.50(0.18)$ & $2.13-2.87$ & $84(49 \%)$ \\
\hline LA size $(\mathrm{cm})$ & $3.35(0.45)$ & $2.61-4.07$ & $3.01(0.05)$ & $2.43-3.67$ & $40(23 \%)$ & $3.18(0.17)$ & $2.61-3.75$ & $28(16 \%)$ \\
\hline LVM (Penn) (g) & $229.8(44.0)$ & $162.7-304.4$ & $143.1(6.3)$ & $112.7-173.5$ & $126(74 \%)$ & $140.0(18.6)$ & $115.6-164.4$ & $135(79 \%)$ \\
\hline LVM (ASE) (g) & $245.7(44.5)$ & $178.0-321.1$ & $148.0(6.3)$ & $116.4-179.7$ & $115(67 \%)$ & $162.1(21.3)$ & $135.8-188.3$ & $113(66 \%)$ \\
\hline
\end{tabular}

*All values significantly greater than normal controls matched for both mean age and body surface area $(p \leqslant 0.001)$.

Ao root, aortic root; ASE, American Society of Echocardiography convention; BSA, body surface area; IVSd, interventricular septal end diastolic dimension; LA size, left atrial end systolic dimension; LVIDd, left ventricular end diastolic internal dimension; LVIDs, left ventricular end systolic internal dimension; LVM, left ventricular mass; LVPWd, left ventricular end diastolic posterior wall dimension; Penn, Penn convention; PSP, adolescent professional soccer players; RVDd, right ventricular end diastolic dimension. 
Table 3 Correlation coefficients between measured echocardiographic variables and age and body surface area of the adolescent professional soccer players

\begin{tabular}{lcclc}
\hline & $\begin{array}{l}\text { Correlation } \\
\text { coefficient: age }\end{array}$ & $p$ Value & $\begin{array}{l}\text { Correlation } \\
\text { coefficient: } B S A\end{array}$ & $p$ Value \\
\hline RVDd & -0.08 & 0.83 & 0.20 & $<0.005$ \\
IVSd & 0.10 & 0.10 & 0.14 & 0.06 \\
LVIDd & 0.14 & 0.04 & 0.36 & $<0.001$ \\
LVPWd & 0.05 & 0.23 & 0.10 & 0.09 \\
LVIDs & 0.14 & 0.04 & 0.26 & $<0.001$ \\
Ao root & 0.08 & 0.13 & 0.28 & $<0.001$ \\
LA size & 0.10 & 0.10 & 0.32 & $<0.001$ \\
LVM (Penn) & 0.21 & $<0.01$ & 0.40 & $<0.001$ \\
LVM (ASE) & 0.20 & $<0.01$ & 0.40 & \\
\hline
\end{tabular}

See table 2 for key to abbreviations.

variables are shown as frequencies and percentages. Comparisons between groups were performed by using paired $t$ tests and associations between age, body surface area, and echocardiographic variables was expressed using simple linear regression calculation of the Pearson product moment and correlation coefficient. All statistical analyses were two sided, and probability values of $\mathrm{p} \leqslant 0.05$ were considered to be significant.

\section{Results}

SUBJECTS

The characteristics of the soccer players are shown in table 1 . We assessed 172 male soccer players over a 32 month period, but one was excluded because of an atrial septal defect requiring surgical closure. The remaining 171 players were white and none had symptoms attributable to cardiovascular disease. No subject was taking any form of prescribed cardiovascular drug treatment at the time of screening. Weekly training varied according to age. In the under 16 years age group the players did 4-5 hours of high intensity training and $2-3$ hours of low intensity training, while in the 16 years and over age group they did 13-15 hours of high intensity training and 15-20 hours of low intensity training.

ECHOCARDIOGRAPHY RESULTS

Table 2 shows the mean, SD, and 5th to 95th centile ranges of the echocardiographic measurements of the soccer player cohort and that predicted for controls matched for age and surface area. Left ventricular dimensions and mass, left atrial chamber size, septal wall thickness, aortic root diameter, and right ventricular diastolic diameter in the soccer players were all significantly increased compared with either age matched or surface area matched control data $(\mathrm{p}<0.001)$.

The ratio of septal wall thickness and posterior wall thickness (IVSd:LVPWd) was always less than 1.5:1. The mean (SD) ejection fraction was $62(7) \%$. No regional wall motion abnormalities of either the right or left ventricles were seen.

Six structural anomalies were discovered incidentally by echocardiography in six soccer players (two bicuspid aortic valves, a haemodynamically insignificant thickened mitral valve, a small patent foramen ovale, a prominent Eustachian valve, and a small atrial septal aneurysm). None of these findings was considered significant enough to warrant further action.
Table 4 ECG changes in adolescent professional soccer players

\begin{tabular}{lcl}
\hline & Mean (SD) & $\begin{array}{l}\text { 5th to 95th centile } \\
\text { range }\end{array}$ \\
\hline Rate (beats/min) & $64(11)$ & 48 to 84 \\
P axis (degrees) & $41(26)$ & -9 to +76 \\
QRS axis (degrees) & $65(28)$ & +13 to +96 \\
T axis (degrees) & $33(17)$ & +6 to +58 \\
PR interval (ms) & $145(21)$ & 112 to 180 \\
QRS duration (ms) & $94(8)$ & 84 to 108 \\
QTc duration (ms) & $402(16)$ & 377 to 428 \\
\hline
\end{tabular}

Table 5 ECG findings in adolescent professional soccer players: criteria suggestive of ventricular hypertrophy and other changes

\begin{tabular}{lc}
\hline & PSP (\%) \\
\hline LVH: Solokow-Lyon & $85(50 \%)$ \\
LVH: Romhilt-Estes score $(\geqslant 5)$ & $29(17 \%)$ \\
RVH: Solokow-Lyon & $1(0.6 \%)$ \\
Repolarisation changes: & \\
$\quad$ Inferior (II,III,AVF) & $15(8.8 \%)$ \\
$\quad$ Anterior (V2-V4) & $2(1.2 \%)$ \\
Lateral (V5-V6) & $2(1.2 \%)$ \\
Incomplete RBBB & $9(5.3 \%)$ \\
Dominant R in V1 & $4(2.3 \%)$ \\
Prominent U waves & $3(1.8 \%)$ \\
P pulmonale & $3(1.8 \%)$ \\
Short PR with no delta wave & $2(1.2 \%)$ \\
Widespread ST elevation & $2(1.2 \%)$ \\
Delta wave & $1(0.6 \%)$ \\
Q waves (V2-V4) & $1(0.6 \%)$ \\
RBBB & $1(0.6 \%)$ \\
\hline
\end{tabular}

$\mathrm{LVH}$, left ventricular hypertrophy; PSP, adolescent professional soccer players; RBBB, right bundle branch block; RVH, right ventricular hypertrophy.

Table 3 shows the results of the correlation analysis between each of the echocardiographic variables and the age and surface area of the soccer players. All variables except IVSd and LVPWd correlated significantly with surface area $(r=0.2$ to $0.4, \mathrm{p}<0.001)$; however, left ventricular mass (American Society of Echocardiographers) was the only variable that correlated with age $(r=0.2, \mathrm{p}<0.01)$.

\section{ECG FINDINGS}

The results of the ECG screening of the soccer players are shown in tables 4 and 5. All soccer players were in sinus rhythm, and sinus bradycardia was present in $65(39 \%)$. None of the players had a higher grade of atrioventricular block than second degree Mobitz type 1 . One player $(0.6 \%)$ had right axis deviation, two $(1.2 \%)$ had left axis deviation, and incomplete right bundle branch block was found in nine $(5.3 \%)$.

Although voltage criteria for right atrial enlargement were found in three subjects $(1.8 \%)$, left atrial enlargement was not seen. Repolarisation changes were seen in 19 players $(11 \%)$, predominantly in the inferior leads. There were no cases of prolonged QTc. ECG changes were not associated with any pathological structural heart disease seen on echocardiography.

Voltage criteria for left ventricular hypertrophy were commonly seen. Eighty five players (50\%) had the Solokow-Lyon voltage criteria for left ventricular hypertrophy, and 29 (17\%) had left ventricular hypertrophy by the Romhilt-Estes points score system. Hypertrophy on both these voltage criteria was compared with echocardiographic hypertrophy 
Table 6 Comparison of echocardiographic left ventricular hypertrophy (LVH) with Solokow-Lyon criteria and Romhilt-Estes score

\begin{tabular}{llc}
\hline LVH criteria & Solokow-Lyon $(\%)$ & $\begin{array}{l}\text { Romhilt-Estes score } \\
(\geqslant 5)(\%)\end{array}$ \\
\hline ECG and echo & 20 & 7 \\
Echo only & 18 & 30 \\
ECG only & 30 & 11 \\
Neither & 32 & 52 \\
\hline
\end{tabular}

(table 6). The Solokow-Lyon criteria were more sensitive, giving a large number of false positives in comparison with the RomhiltEstes points score system, which was too specific and less sensitive. Overall, the ECG criteria did not correlate well with the echocardiographic criteria of left ventricular hypertrophy. Right ventricular hypertrophy was also assessed but was rarely seen.

\section{Discussion}

We have shown that, as with adult professional soccer players, there are significant increases in chamber dimensions, wall thickness, left ventricular mass, and aortic root size in adolescent professional players compared with the values predicted for age and surface area matched controls. Sinus bradycardia and the ECG criteria of left ventricular hypertrophy were common, but the latter correlated poorly with echocardiographic left ventricular hypertrophy.

\section{ECHOCARDIOGRAPHIC CHANGES}

The morphological changes within the heart induced by habitual training consist of an increase in ventricular chamber size, wall thickness, and mass, all of which can be determined by echocardiography. Broadly, two different structural forms of athlete's heart can be defined, depending on the predominant type of exercise undertaken. ${ }^{2}$ If the exercise is mainly isotonic - as in endurance sports such as long distance running - the heart is required to adapt to the volume overload associated with the exceptionally large increases in cardiac output during activity. Thus the left ventricular chamber size increases and there is a proportional increase in wall thickness (eccentric hypertrophy). However, in strength trained athletes who are involved in predominantly isometric exercise (for example, weight lifters, body builders, and wrestlers), increased wall thickness develops but with a relatively unchanged chamber size (concentric hypertrophy). The reason for this seems to be pressure overload produced by the very high systemic arterial pressures generated during their sports. Neither form of hypertrophy appears to be deleterious in the absence of pathological heart disease, as it will regress rapidly with the discontinuation of training. ${ }^{19}$ Longer term studies of athletes have also shown ECG evidence of regression. ${ }^{2021}$

Some sports involve combined endurance and strength training (for example, cycling and rowing) and would be expected to show intermediate changes. This has been confirmed by a large study of athletes' hearts carried out by Spirito and colleagues, studying athletes representing 27 different sports. ${ }^{4}$ They ranked the disciplines according to the changes induced on left ventricular diastolic cavity dimension and left ventricular wall thickness, and found that cyclists had the largest increases in cavity dimension (with rowers seventh), and the rowers had the greatest increase in left ventricular wall thickness (with cyclists second). These large increases in both chamber size and wall thickness are not unexpected, owing to the combination of extreme pressure and volume loading produced during exercise. Indeed during rowing the heart rate can increase to near maximum values of 190 beats/min, with peak systolic blood pressure often exceeding $200 \mathrm{~mm} \mathrm{Hg}^{22}$

Our results showed increases in left ventricular chamber size and wall thickness as well as in left ventricular mass, suggesting a pattern of hypertrophy closer to a combined endurance and strength trained heart than either a purely endurance trained or a purely strength trained heart. This is reflected in the nature of the exercise undertaken by the soccer players, which combines repetitive pressure and volume loading from isometric and isotonic exercise. In a meta-analysis of studies involving elite adult male athletes engaging in purely dynamic, purely static, and combined dynamic and static sports, Pluim and colleagues confirmed the existence of divergent forms of cardiac adaptation compared with control subjects. ${ }^{5}$ A direct comparison between the soccer players and adult athletes included in the meta-analysis did not adjust for differing body size; however, relative wall thickness-calculated as a ratio between the sum of posterior wall and interventricular septum thicknesses and left ventricular cavity dimension - is dimensionless and not dependent on body size. This calculation gives a mean (SD) relative wall thickness of 0.397 (0.059) for the soccer players, which is close to the mean value for combined endurance and strength trained athletes in the metaanalysis (0.398).

Interestingly there was no difference in left ventricular systolic function between the adult athletes of any discipline and the control subjects, a finding replicated in our study. This is further evidence that there is no relation between cardiac morphology and systolic function in the athlete's heart. Left ventricular diastolic function was not assessed in our study.

\section{ECG FINDINGS}

ECG changes in the athlete are also common. ${ }^{6}$ These consist of a high prevalence of sinus bradycardia and arrhythmia, and prolongation of the PR, QRS, and corrected QT intervals compared with control subjects. Sinus pauses of more than two seconds, junctional escape beats, and varying degrees of AV block have been described in athletes and are thought to be caused by increased vagal tone, but usually disappear with exercise or increased sympathetic tone. In addition, the Solokow voltage criteria and the Romhilt-Estes points score often suggest left ventricular hypertrophy, and there may be non-specific $T$ wave inversion in the inferior leads. The criteria for left and right 
atrial enlargement and right ventricular hypertrophy are also often encountered. An especially common pattern found in athletes is the presence of early repolarisation changes, with bizarre $\mathrm{T}$ waves in the anterior leads (juvenile $\mathrm{T}$ wave pattern). However, it is unusual for athletes to have the voltage criteria of left ventricular hypertrophy together with downsloping ST-T changes (strain pattern) or significant left axis deviation and deep pathological $Q$ waves. $^{23}$ These training induced ECG changes are thought to reflect alterations in autonomic tone and structural changes within the myocardium. ${ }^{24}$

In a study by Sharma and colleagues of 1000 highly trained junior athletes with a mean age of 16 years, the Solokow criteria and the Romhilt-Estes points score for left ventricular hypertrophy were found in $45 \%$ and $10 \%$ of the subjects, respectively. ${ }^{25}$ No athlete had left axis deviation, ST segment depression, deep T wave inversion, or pathological $Q$ waves indicative of pathological hypertrophy. However, echocardiography was not performed to quantify the presence or absence of hypertrophy more accurately. This is important because, although the Solokow criteria for left ventricular hypertrophy correlated poorly with the echocardiographic findings, ${ }^{26}$ the more exacting Romhilt-Estes points score correlated better. $^{27}$ In addition, although their study included 310 soccer players, no distinction was made between the nine different sports and the effects of different types of training.

Our ECG findings in soccer players showed a slightly higher incidence of left ventricular hypertrophy $(50 \%$ with the Solokow criteria and $17 \%$ with the Romhilt-Estes points score of 5 or more). However, the correlation between echocardiographic left ventricular hypertrophy and either of these criteria was still poor, so assessing left ventricular hypertrophy in athletes using these indices has little clinical value. Although two soccer players had ST-T changes in the lateral leads simulating the strain pattern, and one had pathological Q waves in his anterior leads, these did not correlate with echocardiographic structural abnormalities or left ventricular hypertrophy.

CLINICAL IMPLICATIONS

Data are available in adults on the differentiation of the athlete's heart from cardiac disease, but such data are lacking in adolescent athletes. The usual criteria for hypertrophic cardiomyopathy include asymmetric septal hypertrophy, septal thickness greater than $16 \mathrm{~mm},{ }^{28}$ small left ventricular cavity size, and systolic anterior motion of the mitral valve apparatus. In dilated cardiomyopathies there is evidence of chamber dilatation (that is, left ventricular end diastolic internal dimension greater than $55 \mathrm{~mm}$ ) together with reduced wall thickness and reduced systolic function. However, the distinction between arrhythmogenic right ventricular dysplasia and athlete's heart may be more difficult, as the two conditions often show both right ventricular dilatation and ECG changes. However, the athletic heart in adults has normal ventricular structure and contractility.
Our observational data on soccer players were compared with ranges predicted for controls matched for age and surface area. Despite the fact that in several of the players the variables measured exceeded the predicted upper limits, no echocardiographic features normally associated with significant heart disease in adults were present. Our reference ranges should help in reducing the diagnostic confusion between physiology and pathology in adolescent athletes, but long term follow up in a larger group may be desirable to see whether those soccer players with echocardiographic or ECG findings outside conventional normal ranges develop heart disease at a later date.

We did find six congenital abnormalities in the soccer players, but this accords with similar age matched epidemiological studies. ${ }^{29}$

PREPARTICIPATION SCREENING

Under intense physical exertion, athletes may die suddenly (sudden cardiac death). The annual incidence of this in male athletes under the age 30 is $1: 200000 .^{30}$ In a necropsy series, Maron and colleagues found that unsuspected inherited or congenital abnormalities accounted for approximately $80 \%$ of deaths, the most common abnormalities being hypertrophic cardiomyopathy (36\%) and congenital malformations of the coronary arteries $(13 \%) .{ }^{31}$ Less commonly, sudden cardiac death has been caused by arrhythmogenic right ventricular dysplasia, myocarditis, premature coronary artery disease, long QT and WolfParkinson-White syndromes, aortic rupture in Marfan's syndrome, mitral valve prolapse, and aortic stenosis. However, a similar series from Italy found a relatively high incidence of arrhythmogenic right ventricular dysplasia in athletes dying suddenly. ${ }^{32}$

In the USA it is advised that all athletes undergo preparticipation cardiovascular screening to identify those with unsuspected cardiovascular problems, and in the UK it has become commonplace over the last few years to screen all soccer players for unsuspected cardiac abnormalities. Such athletes are usually disqualified from competition to reduce their risk of future injury and death.

It has been recommended that preparticipation cardiovascular screening should be confined to a thorough history and physical examination, ${ }^{33}$ but non-invasive testing using a 12 lead ECG and transthoracic echocardiography has been found to enhance the diagnostic yield further. ${ }^{34}$ However, the widespread use of noninvasive testing as part of a mass screening strategy of all athletes of all grades, disciplines, and ages would probably result in an unacceptably high level of false positive results and would not be cost-effective. A more selective policy of only screening individuals with specific cardiovascular symptoms or those who have a family history of sudden cardiac death would probably be a better approach to adopt. This, coupled with the judicious use of non-invasive tests and screening only those elite athletes involved in intensive competition and training, would unearth more potentially life threatening conditions. It is therefore 
imperative that the range of normality for individual groups of sportsmen and women is properly defined, to allow true pathological conditions to be distinguished from the morphological changes associated with intensive physical training.

\section{LIMITATIONS OF THIS STUDY}

In our study we used regression equations derived from normal subjects to obtain control data matched for age and surface area, rather than using specific controls matched for body size. In a study by Huwez and colleagues, ${ }^{13}$ the best correlation observed was between body surface area and left ventricular mass $(r=0.89)$, while the worst was between age and interventricular septal end diastolic dimension $(r=0.44)$. However, not all the individual correlation coefficients for each echocardiographic variable were stated, making it difficult to assess the value of the regression equations for prediction. Given the physical nature of soccer and the intensity of the training regimen, it is not surprising that the soccer players were of a greater than average body size, thus making it difficult to select suitable sedentary controls. The calculated normal ranges that we employed used both body surface area and age as dependent variables to reduce the chance of any error induced by differences in growth and development at various ages. The use of relative wall thickness also adjusts for differences in body size. Unfortunately our correlation analysis between the echocardiographic variables and the age and body surface area of the soccer players did not allow the upper and lower limits of $M$ mode echo measurements to be defined (the prediction interval would have been too large for clinical application).

CONCLUSIONS

Our results show that cardiac dimensions, left ventricular wall thickness and mass, and aortic root size are significantly greater in soccer players than in a reference population matched for age and body surface area. There were no features of pathological heart disease; in particular there was no reduction in left ventricular systolic function. The mean relative wall thickness suggested a pattern of cardiac hypertrophy in keeping with a mixture of isometric and isotonic exercise. This would be typical of a combined endurance and strength trained sport such as soccer. ECG analysis showed that sinus bradycardia was common, as were two different sets of ECG criteria for left ventricular hypertrophy. However, the ECG criteria of left ventricular hypertrophy correlated poorly with echocardiographic findings. Repolarisation changes were also often seen.

We have defined a new set of echocardiographic and ECG reference ranges for soccer players. This information may help to prevent ECG and echocardiographic changes in soccer players being erroneously attributed to disease.

We are indebted to the cardiac technicians at the Cardiothoracic Centre, Liverpool and the six professional football teams in the north west of England for their help and cooperation in the collection of the data.
1 Huston TP, Puffer JC, Rodney WM. The athletic heart syndrome. N Engl F Med 1985;313:24-32.

2 Morganroth J, Maron BJ, Henry WL, et al. Comparative left ventricular dimensions in trained athletes. Ann Intern Med 1975;82:521-4

3 Maron B. Structural features of the athlete's heart as defined by echocardiography. F Am Coll Cardiol 1986;7:190-203.

4 Spirito P, Pellicia A, Proschan MA, et al. Morphology of the "athlete's heart" assessed by echocardiography in 947 elite athletes representing 27 sports. Am F Cardiol 1994;74:802-6.

5 Pluim BM, Zwinderman AH, van der Laarse A, et al. The athlete's heart: a meta-analysis of cardiac structure and function. Circulation 1999;100:336-44.

6 Zehender P, Meinertz T, Keul J, et al. ECG variants and cardiac arrhythmias in athletes: clinical relevance and prognostic importance. Am Heart $\mathcal{f}$ 1990;119:1378-91.

7 Muir DF, MacGregor GD, McCann GP, Hillis WS. The prevalence of LVH in elite professional footballers. Int $\mathcal{f}$ prevalence of LVH in el
Cardiol 1999;71:129-34.

8 DuBois D, DuBois EF. A formula to estimate approximate surface area if height and weight be known. Arch Intern Med 1916;17:863-71.

9 Sahn DJ, DeMaria A, Kislo J, et al. Recommendations regarding quantitation in M-mode echocardiography: results of a survey of echocardiographic measurements. Circulation 1978;58:1072-83.

10 Schiller NB, Shah P, Crawford M, et al. Recommendations for quantitation of the left ventricle by two-dimensional echocardiography. f Am Soc Echocardiogr 1989;5:358-67.

11 Devereux RB, Reichek N. Echocardiographic determination of left ventricular mass in man. Circulation 1977;55:61318.

12 Troy BL, Pombo J, Rackley CE. Measurement of left ventricular wall thickness and mass by echocardiography. Circulation 1972;45:602-11.

13 Huwez FU, Houston AB, Watson J, et al. Age and body surface area related normal upper and lower limits of $M$ mode echocardiographic measurements and left ventricular volume and mass from infancy to early adulthood. Br Heart 1994;72:276-80.

14 Bazett HC. An analysis of the time relations of electrocardiograms. Heart 1920;7:353-67.

5 Morris JJ, Estes EH, Whalen RE, et al. P-wave analysis in valvular heart disease. Circulation 1964;29:242.

16 Solokow M, Lyon P. The ventricular complex in left ventricular hypertrophy as obtained by unipolar precordial and limb leads. Am F Cardiol 1949;37:161-8.

17 Romhilt DW, Estes EH. A point-score system for the ECG diagnosis of left ventricular hypertrophy. Am Heart f 1968; 75:752-8.

18 Solokow M, Lyon P. The ventricular complex in right ventricular hypertrophy as obtained by unipolar precordial ventricular hypertrophy as obtained by unipol

19 Fagard R, Aubert A, Lysens R, et al. Non-invasive Fagard R, Aubert A, Lysens R, et al. Non-invasive
assessment of seasonal variations in cardiac structure and function in cyclists. Circulation 1983;67:896-901.

20 Murrayama M, Kuroda Y. Cardiovascular future in athletes. In: Lubich T, Venerando A, eds. Sports cardiology. Bologna: Aulo Gaggi, 1980:401-13.

21 Dickhuth HH, Hortstmann T, Staiger J, et al. The long-term involution of physiological cardiomegaly and cardiac hypertrophy. Med Sci Sports Exerc 1989;21:244-9.

22 Clifford PS, Hanel B, Seher NH. Arterial blood pressure response to rowing. Med Sci Sports Exerc 1994;26:715-19.

23 Oakley DG, Oakley CM. The significance of abnormal electrocardiograms in highly trained athletes. Am $\mathcal{F}$ Cardiol 1982;50:985-9. 24 Zeppilli P, Pirrami MM, Sassara M, et al. T wave enol, atropine, and exercise. Am Heart f 1980;100:213-22.

25 Sharma S, Whyte G, Elliott P, et al. Electrocardiographic changes in 1000 highly trained junior elite athletes. $\mathrm{Br} F$ Sports Med 1999;33:319-24.

26 Raskoff WJ, Goldman S, Cohn K. "The athletic heart". Prevalence and physiological significance of left ventricular enlargement in distance runners. $\mathcal{F} A M A$ 1976;236:158-62.

27 Kansal S, Roitman DI, Sheffield LT. A quantitative relationship of electrocardiographic criteria of left ventricular hypertrophy with echocardiographic left ventricular mass. A multivariate approach. Clin Cardiol 1983;6:456-63.

28 Pelliccia A, Maron BJ, Spataro A, et al. The upper limit of physiologic cardiac hypertrophy in highly trained athletes. Engl f Med 1991;324:295-301.

29 Feldt RH, Avasthey P, Yoshimasu F, et al. Incidence of congenital heart disease in children born to residents of Olmsted County, Minnesota, 1950-1969. Mayo Clin Proc 1971; 46:794-9.

30 Maron BJ, Gohman TE. Prevalence of sudden cardiac death during competitive sports activities in Minnesota high school athletes. F Am Coll Cardiol 1998;32:1881-4.

31 Maron BJ, Shirani J, Poliac LC, et al. Sudden death in young competitive athletes. Clinical, demographic, and pathological profiles. $\mathcal{F} A M A$ 1996;276:199-204.

32 Corrado D, Thiene G, Nava A, et al. Sudden death in young competitive athletes. Clinical pathologic correlations in 22 cases. Am f Med 1990;89:588-96.

33 Maron BJ, Thompson PD, Puffer JC, et al. Cardiovascular preparticipation screening of competitive athletes. A state-
ment for health professionals from the sudden death comment for health professionals from the sudden death com-
mittee (clinical cardiology) and congenital cardiac defects mittee (clinical cardiology) and congenital cardiac defects committee (cardiovascular disease in the young),

34 Rich BS. Sudden death Screening. Med Clin North Am 1994;78:267-88. 